OPEN ACCESS

Edited by: Elizabeth J. Ryan, St Vincent's University Hospital, Ireland

Reviewed by: Carmen Guerra, Centro Nacional De Investigaciones

Oncologicas, Spain Paola Martinelli, Medical University Vienna, Austria

*Correspondence: Andrew Eugene Hendifar andrew.hendifar@cshs.org

Specialty section:

This article was submitted to Gastrointestinal Cancers, a section of the journal

Frontiers in Oncology

Received: 26 February 2016 Accepted: 11 April 2016 Published: 25 April 2016

Citation:

Iriana S, Ahmed S, Gong J, Annamalai $A A$, Tuli $R$ and Hendifar $A E$ (2016) Targeting mTOR in Pancreatic Ductal Adenocarcinoma. Front. Oncol. 6:99. doi: 10.3389/fonc.2016.00099

\section{Targeting mTOR in Pancreatic Ductal Adenocarcinoma}

\author{
Sentia Iriana ${ }^{1}$, Shahzad Ahmed ${ }^{1}$, Jun Gong ${ }^{1,2}$, Alagappan Anand Annamalai ${ }^{3}$, Richard Tuli \\ and Andrew Eugene Hendifar ${ }^{5 *}$ \\ ${ }^{1}$ Department of Medicine, Cedars-Sinai Medical Center, Los Angeles, CA, USA, ${ }^{2}$ Department of Medical Oncology, City of \\ Hope National Medical Center, Duarte, CA, USA, ${ }^{3}$ Department of Surgery, Cedars-Sinai Medical Center, Los Angeles, CA, \\ USA, ${ }^{4}$ Department of Radiation Oncology, Cedars-Sinai Medical Center, Los Angeles, CA, USA, ${ }^{5}$ Department of Medicine, \\ Division of Medical Oncology, Cedars-Sinai Medical Center, Los Angeles, CA, USA
}

Treatment options for advanced pancreatic ductal adenocarcinoma (PDAC) are limited; however, new therapies targeting specific tumor-related molecular characteristics may help certain patient cohorts. Emerging preclinical data have shown that inhibition of mammalian target of rapamycin (mTOR) in specific KRAS-dependent PDAC subtypes leads to inhibition of tumorigenesis in vitro and in vivo. Early phase II studies of monomTOR inhibition have not shown promise. However, studies have shown that combined inhibition of multiple steps along the mTOR signaling pathway may lead to sustained responses by targeting mechanisms of tumor resistance. Coordinated inhibition of mTOR along with specific KRAS-dependent mutations in molecularly defined PDAC subpopulations may offer a viable alternative for treatment in the future.

Keywords: pancreatic ductal adenocarcinoma, mTOR, KRAS, preclinical, clinical trials

\section{INTRODUCTION}

\section{Epidemiology}

Pancreatic ductal adenocarcinoma (PDAC) remains the fourth leading cause of cancer-related mortality throughout the United States, with 12.3 new cases reported per 100,000 men and women annually and 10.9 deaths reported per 100,000 men and women annually (1). The lifetime risk of developing pancreatic cancer is approximately $1.5 \%$ among men and women, with about $6.7 \%$ survival rate 5 years from diagnosis (1).

Pancreatic ductal adenocarcinoma is associated with poor prognosis. This is related to lack of standardized preventive screening, advanced age during diagnosis with median age being 71 years old, and advanced stage during diagnosis which has allowed only $15-20 \%$ to be surgically resectable at the time of presentation (2). These factors, and the aggressive nature of PDAC, has significantly limited the success of current treatment options, yielding continued low survival rates even in cases which are amenable to surgical resection, modest responses to chemotherapy and radiation, as well as development of resistance to such therapies $(2,3)$.

\section{Standards of Treatment}

Resectability of PDAC is determined clinically by the patients underlying comorbidities and ability to tolerate major surgery, and radiographically based on involvement of the surrounding major vasculature including the superior mesenteric vein and artery, portal vein, celiac artery, and its branches, including the hepatic artery $(2,4)$. For resectable disease (stage I or II), pancreaticoduodenectomy is performed for tumors involving the head and uncinate of the pancreas, whereas distal pancreatectomy 
is performed for tumors of the body and tail and are considered potentially curative (2). However, only $15-20 \%$ of the patients are considered surgical candidates with many of them found to have microscopically positive margins (R1) discovered after surgery on final pathological review $(2,5)$, and others are considered medically unfit often secondary to malnutrition or advanced age and would not benefit from surgical resection or may experience major complications after pancreatic resection $(6,7)$.

Adjuvant (postoperative) therapy with the intention of reducing locoregional and metastatic recurrence has been shown to improve survival compared to postoperative observation alone (6). The Charité Onkologie (CONKO)-001 trial and the European Study Group for Pancreatic Cancer (ESPAC)-3 trial have investigated and established gemcitabine or 5-fluorouracil (FU) as effective avenues for adjuvant chemotherapy compared to observation (6). Neoadjuvant and adjuvant combination chemotherapy including fluorouracil, irinotecan, oxaliplatin, and leucovorin (FOLFIRINOX) and gemcitabine plus albumin-bound paclitaxel particles (nab-paclitaxel) is still being investigated, and adjuvant radiation has demonstrated mixed results $(3,6)$.

Stage III disease is divided into borderline resectable disease $\left[<180^{\circ}\right.$ contact with the superior mesenteric artery (SMA) and locally advanced, unresectable disease $\left(>180^{\circ}\right.$ contact with SMA)] (6). Neoadjuvant (preoperative) therapy is often recommended for borderline resectable disease to address the high probability of positive margins at resection (6). The combination of FOLFIRINOX and gemcitabine + nab-paclitaxel for the treatment of Stage III locally advanced unresectable tumors has limited data, but is commonly used. Again, adjuvant radiation is not adequately supported (2).

Curative resection is not recommended in stage IV PDAC, and treatment focuses on palliation. The use of FOLFIRINOX or gemcitabine + nab-paclitaxel has been shown to extend survival by at least 2 years in at least $10 \%$ of the patients, survival numbers that were rarely seen before and now represent standard first-line options, particularly in patients with good performance status [ECOG 0-1 $(2,8)$ ]. Overall, multidisciplinary, symptomatic, and supportive therapies play an integral role in management (6).

\section{Rationale for mTOR Pathway}

The investigation of genetic and molecular characteristics of PDAC remains a focus of current innovations in an effort to identify potential therapeutic targets. Genomic analysis of PDAC has revealed complex mutational patterns including near ubiquitious activation of KRAS, inactivation of $>50 \%$ of major genetic pathways, such as TP53, SMAD4, and CDKN2A, involvement of $10 \%$ of genes in chromatin modification and DNA damage repair, and a collection of infrequently mutated genes that contribute to heterogeneity and create challenges in the development of targeted therapies (9). Affected gene mutations known to be important in pancreatic cancer turmorigenesis include TP52, SMAD4, CDKN2A, ARID1A, and ROBO2. In one evaluation, utilizing whole genome sequencing and copy number variation of PDAC, combining structural variation events with deleterious point mutations increased the prevalence of inactivation events to $74 \%$ for TP53, 31\% for SMAD4, and 35\% for CDKN2A (9).
The KRAS proto-oncogene is mutated in $90 \%$ of PDAC, with somatic alterations and locally rearranged, focal amplifications being quite common $(6,9,10)$. Single point mutations in codon $12,13,59$, or 61 of exon 2 and exon 3 of the KRAS oncogene impair intrinsic GTPase activity of KRAS and lead to a permanent active KRAS signaling pathway, resulting in proliferation and survival of cells (11). Mutation in KRAS leads to the uncontrolled activation of downstream intracellular signaling pathways such as the RAF/MEK/extracellular signal regulated kinase (ERK) and AKT contributing to tumor cell proliferation and survival $(6,12)$.

While wild-type KRAS has been described as a predictive marker for treatment success of EGFR, inhibitors such as erlotinib or cetuximab and panitumumab in metastatic non-small cell lung and colorectal cancer, its predictive and prognostic value in PDAC has not been clearly established (13). Inhibitors of KRAS have been largely unsuccessful in clinical trials and emphasis has been placed on its downstream pathways (6).

Additional downstream players of KRAS include phosphatidylinositol 3-kinase (PI3K) and AKT, which link ligation of growth factor receptors to the phosphorylation and activation of the serine/threonine kinase, mammalian target of rapamycin (mTOR), further downstream $(14,15)$. This downstream effector plays a role in cell survival, growth, proliferation, and motility, as well as a regulation of apoptosis $(14,15)$. mTOR exists as two complexes: mTORC1 that is rapamycin sensitive and mTORC2 that is largely rapamycin insensitive. mTORC1 interacts with the accessory protein Raptor-to-phosphorylate effectors S6 kinase 1, which ultimately enhances the translation of mRNAs, including ribosomal proteins, elongation factors, and insulin growth factor 2 (14). mTORC1 also phosphorylates $4 \mathrm{EBP} 1$ promoting dissociation of eIF4E from 4EBP1, thus relieving the inhibitory effect of $4 \mathrm{EBP} 1$ on eIF4E-dependent translation initiation, which again ultimately leads to increased translation of mRNAs (14). mTORC2 interacts with its companion RICTOR to phosphorylate PKC alpha and AKT contributing to cell survival, migration, and regulation of the actin cytoskeleton (14).

The mTOR complex is also closely related to the insulin/IGF-1 pathway. Decrease of adenosine triphosphate (ATP) production by metformin, for example, leads to AMPK activation and disruption of insulin/IGF-1 signaling through inhibition of mammalian target of rapamycin $(8,16)$. Inhibition of mTOR signaling, in turn, results in decreased protein synthesis and cell growth. Metformin can also inhibit mTOR signaling through activation of AMPK-independent pathways, including Rag GTPase (17) and REDD1 (18). AMPK-induced activation of tumor suppressor 53 (p53) and subsequent cell cycle arrest represents another potential mechanism of action of metformin in pancreatic cancer models (19). Clearly, the mTOR pathway is a key player in many biological processes including cell growth, regulation of actin cytoskeleton, gene transcription, ribosome biogenesis, mRNA translation, and cell survival and proliferation (14).

Upstream of mTOR, the PI3K/AKT pathway is influenced by PTEN, the negative regulator of PI3K signaling, which decreases its expression in many cancers including pancreatic, and may be downregulated through several mechanisms including mutation, deletion, and methylation (15). 
Clinically investigated mTOR inhibitors include rapamycin (sirolimus) and its analogs, such as temsirolimus, everolimus, and ridaforolimus. These analogs combine with mTOR accessory protein FKBP12 forming complexes that bind to mTOR and inhibit mTORC1 downstream signaling, preventing S6K1 and 4EBP1 phosphorylation (14). While FKBP12-rapamycin complex cannot bind directly to mTORC2, prolonged treatments can disturb mTORC2 assembly and inhibit the phosphorylation of its downstream substrate AKT (14). However, inhibition of mTORC1 without mTORC2 inhibition may stimulate tyrosine kinase activity leading to AKT upregulation, a feedback loop that has been thought to contribute to mTOR resistance. Compared to rapamycin and its analogs, agents that were able to inhibit mTORC1 and mTORC2 were more effective in preclinical evaluations (20). These inhibitors have already been demonstrated to be promising therapeutic agents in other types of malignancies. A high response rate was observed with everolimus in Phase II trials in Hodgkin lymphoma, non-Hodgkin's lymphoma, and breast cancer, and temsirolimus in Phase II/III trials in endometrial cancer and mantle-cell lymphoma (14).

\section{PRECLINICAL STUDIES}

\section{In Vitro mTOR Inhibitor Studies}

In vitro studies have demonstrated diverse effects of mTOR inhibition on cell cycle arrest, autophagy, decreased desmoplastic inflammation, and inhibited epithelial-to-mesenchymal transition in preclinical studies of pancreatic cancer (Table 1).

TABLE 1 | The preclinical development of mTOR inhibitors in pancreatic cancer.

\begin{tabular}{|c|c|c|}
\hline Study agent & $\begin{array}{l}\text { Source in which antitumor } \\
\text { activity was demonstrated }\end{array}$ & Reference \\
\hline Rapamycin & $\begin{array}{l}\text { BxPC3, Su86.86, HS700T, HPAF, } \\
\text { and Capan-1 cells (in vitro) }\end{array}$ & (21) \\
\hline Rapamycin & PANC-1 cells (in vitro) & (24) \\
\hline INK-128 & $\begin{array}{l}\text { Primary human PDAC, PANC-1, } \\
\text { and MIA PaCa-2 cells (in vitro) }\end{array}$ & (25) \\
\hline $\begin{array}{l}\text { BEZ235 } \pm \text { PD0325901, } \\
\text { PKI-587 } \pm \text { PD0325901, and } \\
\text { GDC-0980 } \pm \text { PD0325901 }\end{array}$ & $\begin{array}{l}\text { MIA PaCa-2 and PANC-1 cells } \\
\text { (in vitro) }\end{array}$ & (26) \\
\hline Rapamycin \pm gemcitabine & $\begin{array}{l}\text { Kras PTEN-deficient mice } \\
\text { model (Pdx1-Cre, KrasG12D/+, } \\
\text { Ptenflox/+, in vivo) }\end{array}$ & (27) \\
\hline AZD8055 \pm erlotinib & $\begin{array}{l}\text { PANC-1 and Capan-1 cells } \\
\text { (in vitro); mouse PANC-1 } \\
\text { xenografts (in vivo) }\end{array}$ & (3) \\
\hline Rapamycin \pm XRT (4 Gy) & PC-2 and PANC-1 cells (in vitro) & (28) \\
\hline INK128 \pm XRT & $\begin{array}{l}\text { PSN1, MIA PaCa-2, and PANC-1 } \\
\text { cells (in vitro), mouse PSN1 } \\
\text { xenografts (in vivo) }\end{array}$ & (29) \\
\hline Rapamycin \pm metformin & Mouse Panc02 xenografts (in vivo) & (30) \\
\hline AZD8055 + BEZ235 & $\begin{array}{l}\text { Kras p53-inactivated mice model } \\
\text { (Pdx1-Cre, LSL-KRASG12D, } \\
\text { p53Lox/+, in vivo) }\end{array}$ & (31) \\
\hline
\end{tabular}

INK-128, mTORC1/2 inhibitor; PD0325901, MEK inhibitor; BEZ235, dual PI3K/mTOR kinase inhibitor; PKI-587, dual PI3K/mTOR kinase inhibitor; GDC-0980, dual PI3K mTOR kinase inhibitor; AZD8055, mTORC1/2 inhibitor; XRT, radiation therapy; Gy, gray.
Rapamycin-induced autophagy and apoptosis in BxPC3, Su86.86, HPAF, Capan-1, and HS700T PDAC cell lines that correlated to cell line-specific levels of mTOR activity (21). Novel agents Alisertib and Plumbagin, induced cell cycle arrest, promoted autophagy, and inhibited epithelial-to-mesenchymal transition in PANC-1 and BxPC3 cell lines through inhibition of PI3K/AKT/ mTOR signaling $(22,23)$.

Mammalian target of rapamycin inhibition with first and second generation inhibitors also leads to class specific mechanisms of resistance. As demonstrated across several cancer cell lines, resistance to mTOR-related cytotoxicity has been limited by feedback activation via the IGF-1R-AKT signaling pathway imparting drug resistance (32). In PANC-1 and MiaPaCa-2 cells, rapamycin treatment leads to resistance mediated by $\mathrm{AKT}$ phosphorylation despite inhibitory effects on proliferation of PANC-1 cells (24). Furthermore, treatment with second generation mTOR inhibitors, such as KU63794 and PP242, leads to treatment resistance via increased ERK activation (26).

Novel mTOR inhibitors may overcome resistance mechanisms by dual inhibition of mTOR complexes. Primary and transformed pancreatic cancer cells exhibit a concentration- and time-dependent arrest of growth upon dual mTOR inhibition with INK-128 via 4E-BP1, S6K1, and AKT (25). Furthermore, INK-128 also sensitizes cells to treatment with gemcitabine. Use of U126 or PD0325901 MEK inhibitors prevents ERK overactivation induced by NPV-BEZ235 (dual PI3K/mTOR kinase inhibitor) leading to synergistic inhibition of proliferation in a dose-dependent manner in PANC-1 and MiaPaCa-2 cells (26).

\section{In Vivo mTOR Inhibition Studies}

Animal models have demonstrated that agents targeting the mTOR pathway can lead to significant inhibition of proliferation, differentiation, and tumor progression in specific PDAC subpopulations (Table 1). Enhanced inhibition of tumor differentiation and progression by rapamycin was demonstrated to be specifically dependent on loss of PTEN in KRAS-mutant mice (KC) (27). Inhibition of mTOR improved survival and induced tumor shrinkage downstream of mTOR via S6 leading to regression of tumors into benign, relatively non-proliferative cysts. In contrast, KRAS-mutant mice tumors driven by mutant p53 (KPC) did not respond to rapamycin treatment, which has other distinct pathways that are mTOR independent $(27,33)$. In transgenic mouse models in which mTOR was hyperactivated either through the KRAS/MEK/ERK cascade, by loss of PTEN, or through TSC1 haploinsufficiency, single inhibition of mTOR or MEK elicited strong feedback activation of ERK or AKT (34). In this study, rapamycin and PD98059 individually lead to ERK and AKT feedback-mediated resistance; however, dual inhibition with LY294002 and PD98059 ameliorated oncogenic activity. Furthermore, PTEN-deficient cells responded to LY294002 and/or rapamycin treatment, but not PD98059, consistent with aforementioned study by Morran and colleagues (27). Analysis of downstream targets in pancreatic cancer cell lines identified that MEK/ERK/TSC/mTOR signaling is dependent on ALDH1A3 function and high expression of ALDH1A3 is associated with an aggressive subtype of PDAC (34). Therefore, in ALDH1A3positive PDAC, targeting of ALDH1A3 may be of benefit in 
addition to inhibiting the MEK/ERK/mTOR cascade. Moreover, PTEN haploinsufficiency also appears to promote tumorigenesis through PI3K-dependent NF- $\mathrm{KB}$ activation in pancreatic cancer mouse models (35). Notably, treatment with LY294002 abrogated $\mathrm{NF}-\kappa \mathrm{B}$ activation in PTEN haploinsufficient pancreatic cancer models in vivo (35).

Use of second generation mTOR inhibitors offers similar distinct mechanisms of tumorigenesis inhibition. AZD8055, a second generation mTOR inhibitor, used with erlotinib (an EGRF inhibitor) leads to proliferative inhibition in PANC-1 xenografts (3). Use of both AZD8055 and erlotinib abolished EGFR/AKT feedback activation-related resistance associated with AZD8055 monotherapy. The combination of AZD8055 and the dual PI3K-mTOR inhibitor BEZ235 delayed PDAC progression and prolonged survival in KRAS-mutant PDAC mice in vivo (31).

\section{mTOR Inhibitors as Radiosensitizers}

First and second generation mTOR inhibitors both act to sensitize PDAC to radiation therapy in vitro (Table 1). PC-2 and PANC-1 cells treated with rapamycin exhibited a dose-dependent radiosensitizing effect on cell proliferation arrest leading to G2/M phase cell cycle arrest (28). PSN1 cells exhibited a dose-dependent inhibition of proliferation and tumor growth delay in athymic nude mice xenografts following single and fractionated doses of radiation with INK-128 pretreatment (29).

\section{Metformin-Related mTOR Inhibition of PDAC}

Metformin exhibits diverse effects on PDAC carcinogenesis through both mTOR-dependent and -independent mechanisms (36). Metformin mTOR activation occurs via AMPK-mediated (16, 37), Rag GTPase-mediated (17), and REDD1-mediated mechanisms (18). Metformin may also ameliorate aberrant signaling and feedback inhibition via insulin-like growth factor-1 receptor (IGF-1R)-AKT signaling by improving insulin tissue sensitivity (16). In MIAPaca2 and PANC1 cells explanted into an athymic nude mice xenograft, metformin inhibits pancreatic cell growth via mTOR1 inhibition, which was demonstrated to be dose dependent (38). Combined treatment with metformin and rapamycin of Panc02 cells transplanted into diet-induced obese (DIO) C57BL/6 mice lead to significantly reduced pancreatic tumor growth and mTOR-related signaling (30).

\section{Inhibition of mTOR in Human PDAC}

In genetic profiles of human tumors, loss of or low PTEN expression and hyperphosphorylation of AKT has been found to be present in around $70 \%$ of the cases and PTEN genomic loss (deletion of one or two copies of the PTEN locus) in 15\% of the cases (35). Even single-copy PTEN loss, in the setting of KRASinitiated pancreatic transformation, retains its tumorigenic potential by increasing epithelial proliferation, contributing to an aggressive histologic phenotype, and activating PI3K/AKT and ERK signaling in vivo (35). Biopsies of human pancreatic cancer cells obtained through endoscopic ultrasonography that overexpressed pS6 (a downsteam effector activated by mTOR overexpression) show sensitivity to rapamycin inhibition ex vivo (21). Recent data examining resected PDAC biology utilizing "Multi-Omic" analysis suggest that alterations in mTOR pathway are very common and potentially important for treatment (39-42). Out of 117 PDAC samples included in one analysis, $43 \%$ of the patients had a therapeutic response related to a molecular abnormality or mechanism/pathway identified through next-generation sequencing (NGS) (41). Actionable findings linked to a specific treatment options identified by NGS included mutations in BRCA2 (5\%), PALB2 (1\%), ATM (4\%), BRAF (2\%), PIK3C/PIK3R (7\%), STK11 (5\%), amplification of ERBB2 (3\%), FGFR (2\%), PDGFR (2\%), and RET fusions (2\%) (42). Further analysis with incorporation of immunohistochemistry (IHC) in this cohort refined and expanded chemotherapy treatment options in all patients (41). A subset of PDAC with integration of phosphoproteomics (PHO) in their NGS and IHC analysis revealed pathway activation (e.g., mTOR, JAK-STAT, MET, RET, or EGFR) in 16/20 samples (41).

Unique genetically driven human PDAC have been reported to respond to targeted mTOR inhibition suggesting that preclinical data are applicable in the treatment of human PDAC. During phase I treatment with MK-2206, a pan-AKT inhibitor, a dramatic $23 \%$ shrinkage in tumor was found in a patient with a loss of PTEN KRAS-dependent PDAC, which was thought to have occurred via PI3K-AKT-mTOR inhibition (43). Similarly, use of everolimus in a patient with Peutz-Jeghers syndrome-induced advanced pancreatic cancer with presumed mTOR hyperactivation through loss of STK11/LKB1 leads to 9 months progressionfree survival (44).

\section{CLINICAL TRIALS}

\section{Clinical Trials Utilizing mTOR Inhibitors}

Rapalogs monotherapy have not been shown to be effective in three phase II clinical trials of gemcitabine-refractory metastatic PDAC. Although treatment was well tolerated in a multi-institutional, single-arm, phase II study of everolimus in patients with gemcitabine-refractory metastatic PDAC, there were no significant improvements in progression-free survival or overall survival (45). The most common grade 3 and 4 treatment-related toxicities were thrombocytopenia and hyperglycemia, respectively, leading to delay in treatment; however, no patients were removed from treatment due to drug-related adverse effects. In an open label, single-arm phase II study in gemcitabine-refractory metastatic PDAC patients treated with either temsirolimus or everolimus/erlotinib, there was similarly no demonstrated improvement in clinical responses (46). Significant systemic toxicity leads to premature cessation of patient enrollment for patients treated with temsirolimus. Treatment with everolimus/erlotinib therapy was tolerated; however, enrollment was also prematurely ended due to progression of disease on therapy.

Although not a direct mTOR inhibitor, MK-2206, an allosteric AKT inhibitor, with selumetinib, a MEK1/MEK2 inhibitor, were included in phase II trial concerning metastatic PDAC 
failing gemcitabine-based therapy. Patients were randomized to treatment groups MK-2206 $135 \mathrm{mg}$ weekly plus selumetinib $100 \mathrm{mg}$ daily (MS) or mFOLFOX6 (without 5-FU bolus) every 2 weeks. The most common toxicities, including rash, mucositis, dehydration, and fatigue, were observed in 34 patients in the MS arm compared to hematologic toxicities, fatigue, nausea, and vomiting observed in 19 patients in the mFOLFOX arm. MS did not improve overall survival, and shorter survival was observed compared to mFOLFOX [median OS 4.0 vs. 7.5 months, hazard ratio (HR) $1.46,95 \%$ CI $0.90-2.38$ ] (47).

Recently, combination therapy with capecitabine (5-FU prodrug) and everolimus in a phase II trial of the first-line and second-line treatment of PDAC demonstrated modest benefit to combined therapy over monotherapy (48). Median overall survival with combination therapy was 12.4 months in first-line patients and 5.9 months in second-line patients compared to historical rates of overall survival with capecitabine monotherapy of 5.9 months in first-line patients and 5.0 months in second-line patients suggesting that addition of everolimus to capecitabine might enhance efficacy of capecitabine monotherapy, especially in first-line patients.

Limitations of the aforementioned trials include the lack of characterization of the molecular pathology underlying PDAC tumorigenesis, which preclinical data suggest would predict treatment response. The modest improvement noted in the combined therapy everolimus/capecitabine phase II study supports the notion that coordinated inhibition of downstream

\section{REFERENCES}

1. Howlader N, Noone A, Krapcho M, Garshell J, Miller D, Altekruse S, et al. SEER Cancer Statistics Review, 1975-2011. (2013). Available from: http://seer. cancer.gov/csr/1975_2011/

2. Ryan DP, Hong TS, Bardeesy N. Pancreatic adenocarcinoma. N Engl J Med (2014) 371:1039-49. doi:10.1056/NEJMra1404198

3. Wei F, Zhang Y, Geng L, Zhang P, Wang G, Liu Y. mTOR inhibition induces EGFR feedback activation in association with its resistance to human pancreatic cancer. Int J Mol Sci (2015) 16:3267-82. doi:10.3390/ijms16023267

4. Chari ST, Leibson CL, Rabe KG, Ransom J, de Andrade M, Petersen GM. Probability of pancreatic cancer following diabetes: a population-based study. Gastroenterology (2005) 129:504-11. doi:10.1016/j.gastro.2005.05.007

5. Konstantinidis IT, Warshaw AL, Allen JN, Blaszkowsky LS, Castillo CF, Deshpande V, et al. Pancreatic ductal adenocarcinoma: is there a survival difference for R1 resections versus locally advanced unresectable tumors? What is a "true" R0 resection? Ann Surg (2013) 257:731-6. doi:10.1097/ SLA.0b013e318263da2f

6. Wolfgang CL, Herman JM, Laheru DA, Klein AP, Erdek MA, Fishman EK, et al. Recent progress in pancreatic cancer. CA Cancer J Clin (2013) 63:318-48. doi:10.3322/caac. 21190

7. Hsu CC, Wolfgang CL, Laheru DA, Pawlik TM, Swartz MJ, Winter JM, et al. Early mortality risk score: identification of poor outcomes following upfront surgery for resectable pancreatic cancer. J Gastrointest Surg (2012) 16:753-61. doi:10.1007/s11605-011-1811-4

8. Gong J, Robbins LA, Lugea A, Waldron RT, Jeon CY, Pandol SJ. Diabetes, pancreatic cancer, and metformin therapy. Front Physiol (2014) 5:426. doi:10.3389/fphys.2014.00426

9. Waddell N, Pajic M, Patch AM, Chang DK, Kassahn KS, Bailey P, et al. Whole genomes redefine the mutational landscape of pancreatic cancer. Nature (2015) 518:495-501. doi:10.1038/nature14169

10. Valero V III, Saunders TJ, He J, Weiss MJ, Cameron JL, Dholakia A, et al. Reliable detection of somatic mutations in fine needle aspirates of pancreatic
KRAS signaling may improve antitumor efficacy of therapy (NCT01337765, NCT01324258, and NCT01562899).

\section{CONCLUSION}

Although initial trials targeting mTOR inhibition have generally failed to demonstrate treatment efficacy, specifically targeting therapy to the diverse mutations underlying KRAS-dependent PDAC in specific subpopulations offer potential for coordinated inhibition of synergistic trophic mechanisms and the resistancerelated feedback mechanisms underlying tumorigenesis. Targeting these mutations offers the advantage of improved tumor treatment with the potential for less systemic toxicity. Inhibition of patient-specific mTOR activity has strong preclinical data. Although many initial clinical trials with mTOR inhibition have been negative, the coordinated inhibition of multiple steps along the mTOR pathway may offer a viable alternative form of treatment for genetically defined PDAC in the future. Results from further late-phase studies involving combined mTOR pathway inhibition are eagerly awaited.

\section{AUTHOR CONTRIBUTIONS}

SI and SA are co-first authors of this paper as they contributed to concept, initial drafting, and literature review. All authors otherwise contributed to this paper through literature review, drafting, critical revision, editing, and final approval of the final version. cancer with next-generation sequencing: implications for surgical management. Ann Surg (2015) 1:1-8. doi:10.1097/sla.0000000000001156

11. Kruger S, Haas M, Ormanns S, Bachmann S, Siveke JT, Kirchner T, et al. Translational research in pancreatic ductal adenocarcinoma: current evidence and future concepts. World J Gastroenterol (2014) 20:10769-77. doi:10.3748/ wjg.v20.i31.10769

12. Arslan C, Yalcin S. Current and future systemic treatment options in metastatic pancreatic cancer. J Gastrointest Oncol (2014) 5:280-95. doi:10.3978/j. issn.2078-6891.2014.030

13. Kim ST, Lim do H, Jang KT, Lim T, Lee J, Choi YL, et al. Impact of KRAS mutations on clinical outcomes in pancreatic cancer patients treated with firstline gemcitabine-based chemotherapy. Mol Cancer Ther (2011) 10:1993-9. doi:10.1158/1535-7163.mct-11-0269

14. Populo H, Lopes JM, Soares P. The mTOR signalling pathway in human cancer. Int J Mol Sci (2012) 13:1886-918. doi:10.3390/ijms13021886

15. Faivre S, Kroemer G, Raymond E. Current development of mTOR inhibitors as anticancer agents. Nat Rev Drug Discov (2006) 5:671-88. doi:10.1038/nrd2062

16. Rozengurt E, Sinnett-Smith J, Kisfalvi K. Crosstalk between insulin/insulin-like growth factor-1 receptors and G protein-coupled receptor signaling systems: a novel target for the antidiabetic drug metformin in pancreatic cancer. Clin Cancer Res (2010) 16:2505-11. doi:10.1158/1078-0432.CCR-09-2229

17. Kalender A, Selvaraj A, Kim SY, Gulati P, Brule S, Viollet B, et al. Metformin, independent of AMPK, inhibits mTORC1 in a rag GTPase-dependent manner. Cell Metab (2010) 11:390-401. doi:10.1016/j.cmet.2010.03.014

18. Ben Sahra I, Laurent K, Loubat A, Giorgetti-Peraldi S, Colosetti P, Auberger $\mathrm{P}$, et al. The antidiabetic drug metformin exerts an antitumoral effect in vitro and in vivo through a decrease of cyclin D1 level. Oncogene (2008) 27:3576-86. doi:10.1038/sj.onc.1211024

19. Jalving M, Gietema JA, Lefrandt JD, de Jong S, Reyners AK, Gans RO, et al. Metformin: taking away the candy for cancer? Eur J Cancer (2010) 46:2369-80. doi:10.1016/j.ejca.2010.06.012

20. Sun SY. mTOR kinase inhibitors as potential cancer therapeutic drugs. Cancer Lett (2013) 340:1-8. doi:10.1016/j.canlet.2013.06.017 
21. Utomo WK, Narayanan V, Biermann K, van Eijck CH, Bruno MJ, Peppelenbosch MP, et al. mTOR is a promising therapeutical target in a subpopulation of pancreatic adenocarcinoma. Cancer Lett (2014) 346:309-17. doi:10.1016/j.canlet.2014.01.014

22. Wang F, Li H, Yan XG, Zhou ZW, Yi ZG, He ZX, et al. Alisertib induces cell cycle arrest and autophagy and suppresses epithelial-to-mesenchymal transition involving PI3K/Akt/mTOR and sirtuin 1-mediated signaling pathways in human pancreatic cancer cells. Drug Des Devel Ther (2015) 9:575-601. doi:10.2147/dddt.s75221

23. Wang F, Wang Q, Zhou ZW, Yu SN, Pan ST, He ZX, et al. Plumbagin induces cell cycle arrest and autophagy and suppresses epithelial to mesenchymal transition involving PI3K/Akt/mTOR-mediated pathway in human pancreatic cancer cells. Drug Des Devel Ther (2015) 9:537-60. doi:10.2147/dddt.s73689

24. Soares HP, Ni Y, Kisfalvi K, Sinnett-Smith J, Rozengurt E. Different patterns of Akt and ERK feedback activation in response to rapamycin, active-site mTOR inhibitors and metformin in pancreatic cancer cells. PLoS One (2013) 8:e57289. doi:10.1371/journal.pone.0057289

25. Lou HZ, Weng XC, Pan HM, Pan Q, Sun P, Liu LL, et al. The novel mTORC1/2 dual inhibitor INK-128 suppresses survival and proliferation of primary and transformed human pancreatic cancer cells. Biochem Biophys Res Commun (2014) 450:973-8. doi:10.1016/j.bbrc.2014.06.081

26. Soares HP, Ming M, Mellon M, Young SH, Han L, Sinnet-Smith J, et al. Dual PI3K/mTOR inhibitors induce rapid over-activation of the MEK/ERK pathway in human pancreatic cancer cells through suppression of mTORC2. Mol Cancer Ther (2015) 14(4):1014-23. doi:10.1158/1535-7163.mct-14-0669

27. Morran DC, Wu J, Jamieson NB, Mrowinska A, Kalna G, Karim SA, et al. Targeting mTOR dependency in pancreatic cancer. Gut (2014) 63:1481-9. doi:10.1136/gutjnl-2013-306202

28. Dai ZJ, Gao J, Kang HF, Ma YG, Ma XB, Lu WF, et al. Targeted inhibition of mammalian target of rapamycin (mTOR) enhances radiosensitivity in pancreatic carcinoma cells. Drug Des Devel Ther (2013) 7:149-59. doi:10.2147/ dddt.s42390

29. Hayman TJ, Wahba A, Rath BH, Bae H, Kramp T, Shankavaram UT, et al. The ATP-competitive mTOR inhibitor INK128 enhances in vitro and in vivo radiosensitivity of pancreatic carcinoma cells. Clin Cancer Res (2014) 20:110-9. doi:10.1158/1078-0432.ccr-13-2136

30. Cifarelli V, Lashinger LM, Devlin KL, Dunlap SM, Huang J, Kaaks R, et al. Metformin and rapamycin reduce pancreatic cancer growth in obese prediabetic mice by distinct microRNA-regulated mechanisms. Diabetes (2015) 64:1632-42. doi:10.2337/db14-1132

31. Alagesan B, Contino G, Guimaraes AR, Corcoran RB, Deshpande V, Wojtkiewicz GR, et al. Combined MEK and PI3K inhibition in a mouse model of pancreatic cancer. Clin Cancer Res (2015) 21:396-404. doi:10.1158/10780432.CCR-14-1591

32. O'Reilly KE, Rojo F, She QB, Solit D, Mills GB, Smith D, et al. mTOR inhibition induces upstream receptor tyrosine kinase signaling and activates Akt. Cancer Res (2006) 66:1500-8. doi:10.1158/0008-5472.can-05-2925

33. Eser S, Reiff N, Messer M, Seidler B, Gottschalk K, Dobler M, et al. Selective requirement of PI3K/PDK1 signaling for Kras oncogene-driven pancreatic cell plasticity and cancer. Cancer Cell (2013) 23:406-20. doi:10.1016/j. ccr.2013.01.023

34. Kong B, Wu W, Cheng T, Schlitter AM, Qian C, Bruns P, et al. A subset of metastatic pancreatic ductal adenocarcinomas depends quantitatively on oncogenic Kras/Mek/Erk-induced hyperactive mTOR signalling. Gut (2015) 0:1-11. doi:10.1136/gutjnl-2014-307616

35. Ying H, Elpek KG, Vinjamoori A, Zimmerman SM, Chu GC, Yan H, et al. PTEN is a major tumor suppressor in pancreatic ductal adenocarcinoma and regulates an NF-kappaB-cytokine network. Cancer Discov (2011) 1:158-69. doi:10.1158/2159-8290.CD-11-0031

36. Rozengurt E. Mechanistic target of rapamycin (mTOR): a point of convergence in the action of insulin/IGF-1 and G protein-coupled receptor agonists in pancreatic cancer cells. Front Physiol (2014) 5:357. doi:10.3389/ fphys.2014.00357

37. El-Mir MY, Nogueira V, Fontaine E, Averet N, Rigoulet M, Leverve X. Dimethylbiguanide inhibits cell respiration via an indirect effect targeted on the respiratory chain complex I. J Biol Chem (2000) 275:223-8. doi:10.1074/ jbc.275.1.223

38. Kisfalvi K, Eibl G, Sinnett-Smith J, Rozengurt E. Metformin disrupts crosstalk between $G$ protein-coupled receptor and insulin receptor signaling systems and inhibits pancreatic cancer growth. Cancer Res (2009) 69:6539-45 doi:10.1158/0008-5472.CAN-09-0418

39. Kristensen VN, Lingjaerde OC, Russnes HG, Vollan HK, Frigessi A, BorresenDale AL. Principles and methods of integrative genomic analyses in cancer. Nat Rev Cancer (2014) 14:299-313. doi:10.1038/nrc3721

40. Basik M, Aguilar-Mahecha A, Rousseau C, Diaz Z, Tejpar S, Spatz A, et al. Biopsies: next-generation biospecimens for tailoring therapy. Nat Rev Clin Oncol (2013) 10:437-50. doi:10.1038/nrclinonc.2013.101

41. Pishvaian MJ, Brody JR, Matrisian L, Hendifar AE, Engebretson A, Hoos WA, et al. Multi-omic profiling (MoP) for patients (pts) with pancreatic cancer (PDA): initial results of the Know Your Tumor (KYT) initiative. J Clin Oncol (2016) 34(Suppl 4S):282.

42. Engebretson A, Brody JR, Rahib L, Matrisian L, Hendifar AE, Hoos WA, et al. The Know Your Tumor (KYT) initiative: a national program of multi-omic molecular profiling (MoP) for patients (Pts) with pancreatic cancer (PDA). J Clin Oncol (2016) 34(Suppl 4S):279.

43. Yap TA, Yan L, Patnaik A, Fearen I, Olmos D, Papadopoulos K, et al. Firstin-man clinical trial of the oral pan-AKT inhibitor MK-2206 in patients with advanced solid tumors. J Clin Oncol (2011) 10:4688-95. doi:10.1200/ JCO.2011.35.5263

44. Klümpen HJ, Queiroz KC, Spek CA, van Noesel CJ, Brink HC, de Leng WW, et al. mTOR inhibitor treatment of pancreatic cancer in a patient with Peutz-Jeghers syndrome. J Clin Oncol (2011) 29:e150-3. doi:10.1200/ JCO.2010.32.7825

45. Wolpin BM, Hezel AF, Abrams T, Blaszkowsky LS, Meyerhardt JA, Chan JA, et al. Oral mTOR inhibitor everolimus in patients with gemcitabine-refractory metastatic pancreatic cancer. JClin Oncol (2009) 27:193-8. doi:10.1200/ jco.2008.18.9514

46. Javle MM, Shroff RT, Xiong H, Varadhachary GA, Fogelman D, Reddy SA, et al. Inhibition of the mammalian target of rapamycin (mTOR) in advanced pancreatic cancer: results of two phase II studies. BMC Cancer (2010) 10:368. doi:10.1186/1471-2407-10-368

47. Chung V, McDonough S, Philip P, Cardin DB, Wang-Gillam A, Hui L, et al. SWOG S1115: randomized phase II trial of selumetinib (AZD6244; ARRY 142886) hydrogen sulfate (NSC-748727) and MK-2206 (NSC-749607) vs. mFOLFOX in pretreated patients with metastatic pancreatic cancer. J Clin Oncol (2015) 33:4119.

48. Kordes S, Klumpen HJ, Weterman MJ, Schellens JH, Richel DJ, Wilmink JW. Phase II study of capecitabine and the oral mTOR inhibitor everolimus in patients with advanced pancreatic cancer. Cancer Chemother Pharmacol (2015) 75:1135-41. doi:10.1007/s00280-015-2730-y

Conflict of Interest Statement: The authors declare that the research was conducted in the absence of any commercial or financial relationships that could be construed as a potential conflict of interest.

Copyright (c) 2016 Iriana, Ahmed, Gong, Annamalai, Tuli and Hendifar. This is an open-access article distributed under the terms of the Creative Commons Attribution License (CC BY). The use, distribution or reproduction in other forums is permitted, provided the original author(s) or licensor are credited and that the original publication in this journal is cited, in accordance with accepted academic practice. No use, distribution or reproduction is permitted which does not comply with these terms. 